\title{
Contextual Bandit Based Rate Adaptation Mechanism for IEEE 802.11 Mobile Wireless LANs
}

This paper was downloaded from TechRxiv (https://www.techrxiv.org).

\section{LICENSE}

CC BY 4.0

SUBMISSION DATE / POSTED DATE

05-12-2021 / 10-12-2021

CITATION

Sen, Arkadeep; Sivalingam, Krishna (2021): Contextual Bandit Based Rate Adaptation Mechanism for IEEE 802.11 Mobile Wireless LANs. TechRxiv. Preprint. https://doi.org/10.36227/techrxiv.17126483.v1

$\mathrm{DOI}$

10.36227/techrxiv.17126483.v1 


\title{
Contextual Bandit Based Rate Adaptation Mechanism for IEEE 802.11 Mobile Wireless LANs
}

\author{
Arkadeep Sen and Krishna M. Sivalingam, Fellow, IEEE
}

\begin{abstract}
Rate adaptation (RA) is used in IEEE 802.11 WLANs to determine the optimal datarate for a particular channel condition. It becomes especially difficult to determine the optimal datarate for the new High-Throughput WLANs (802.11ac/ax) since the number of available datarates in these standards are very high. Moreover, a mobile environment poses additional challenge in RA as the channel conditions will keep on changing from time to time. In this paper, we propose a Contextual Bandits based Rate Adaptation (ContRA) algorithm for mobile users in IEEE 802.11ac/ax standards. Based on the Received Signal Strength Indicator (RSSI) range that the receiver is currently in, the RA algorithm tries to determine the optimal rate from the rate set suitable for packet transmission in that RSSI range. Performance studies show that the proposed RA algorithm is able to adapt to changing channel conditions and quickly choose a suitable datarate for those channel conditions.
\end{abstract}

Index Terms-Wireless Local Area Network, Rate Adaptation, Contextual Bandits.

\section{INTRODUCTION}

$\mathbf{R}$ ATE adaptation is an important aspect of IEEE 802.11 Wireless Local Area Networks (WLANs) since it helps in maximizing the throughput under varying wireless channel conditions. Rate adaptation is used to determine the optimal datarate that can yield the maximum throughput. Choosing a non-optimal datarate for a particular channel condition will reduce the overall throughput achieved by the WLAN. Moreover, it may result in high packet loss as well.

The number of available datarates has increased manyfold from that of the older IEEE 802.11 standards to that of the recent High-Throughput WLANs (HT-WLANs). As the number of datarates increases, it becomes more challenging to select an optimal rate for a particular channel condition. In a mobile environment, the channel conditions can often change, making it even more difficult to adapt. As a result, the rate adaptation algorithms used for older IEEE 802.11 standards will not work efficiently, or may even completely fail, to determine the optimal datarate for HT-WLANs.

Many previous works [1]-[4] have used Multi-armed Bandit (MAB) [5] problem to model rate adaptation in HT-WLANs. MAB is a classic Reinforcement Learning (RL) problem, in which an agent takes an action in every timestep and gets a

The authors are with the Department of Computer Science and Engineering, Indian Institute of Technology Madras, Chennai, India (Email: arkadeep.sen87@gmail.com; skrishnam@cse.iitm.ac.in, krishna.sivalingam@gmail.com).

A preliminary version of this paper has been accepted as a SHORT 4-page paper at IEEE LCN 2020 conference.

This work was supported by a Mid-Career Institute Research and Development Award (IRDA) from IIT Madras (2017-2020), by a DST grant (EMR/2016/003016) from Government of India (2017-2020). reward for the action taken. However, these works either do not take into account the effect of mobility on the channel conditions, or do not directly incorporate the variation of the channel conditions in the model. For different channel conditions, the optimal rate for maximizing throughput may be different. As shown in [6], [7], certain datarates work well for a range of Received Signal Strength Indicator (RSSI) values. However, for other RSSI ranges, using the same datarates may lead to reduced throughput and high packet loss. Thus, it is important to estimate the RSSI range that the receiver is in and then find out the optimal datarate from the set of datarates suitable for packet transmission for that particular range. It is difficult to model such a behavior using only MAB.

In [8], we proposed the use of Contextual Bandit problem, which is an extension of MAB, to model the rate adaptation in a mobile environment. In this paper, we have extended the work by: (i) proposing two variants of the original algorithm - as described in Section III; (ii) developing the model for IEEE 802.11ax standard; (iii) adding performance results for scenarios where the user moves away from the Access Point; and (iv) adding performance results for multiple users communicating using a given AP.

In the Contextual Bandits setting [5], [9], [10], the agent gets a context in every timestep before it takes an action. The action set and the reward distribution of the actions may vary for different contexts. The goal of an agent, in a Contextual Bandit setting, is to maximize the total reward over the timesteps by identifying the optimal action for each context. In particular, we present a Contextual Bandits based Rate Adaptation (ContRA) algorithm for mobile users in IEEE 802.11ac/ax standards [11], [12]. The ContRA algorithm is designed to achieve the following goals:

(i) Determine datarate based on the RSSI range the receiver is in: Before a packet is sent to a receiver, the sender will first estimate the RSSI range in which the receiver is in. Then, the rate adaptation algorithm will try to ascertain the optimal rate for that RSSI range to maximize the overall throughput. The RSSI range can be directly incorporated in the ContRA algorithm as the context, which is not possible in an MAB-based RA algorithm.

(ii) Reuse information already learnt: The sender will run only one instance of the ContRA algorithm for all the receivers as opposed to running separate instances of the algorithm for each receiver. Thus, the sender can use the prior information, learnt from the packet transmissions of the previous receivers, for a new receiver in a particular RSSI range. As a result, the optimal rates for the different RSSI ranges can be determined 
quickly.

(iii) Use datarate set suitable for a particular RSSI range: For each RSSI range, the set of suitable datarates for that range are used instead of all the available datarates. Since RSSI range is used as the context, different datarate sets for different RSSI ranges (contexts) can be easily specified in the ContRA algorithm. As a result, the rate adaptation algorithm can quickly determine the optimal datarate for an RSSI range without sending packets using highly sub-optimal datarates which are unsuitable for that RSSI range. This avoids packet transmissions with high loss and low throughput, and ultimately maximizes the overall throughput.

The proposed ContRA algorithm has been implemented in ns3 network simulator [13] for performance evaluation. The results show that the proposed ContRA algorithm is able to adapt to the changing channel conditions and achieve high throughput for both UDP and TCP applications.

\section{BACKGROUND AND RELATED WORK}

This section presents the relevant background and related work.

\section{A. Wireless Local Area Networks (WLANs)}

In a wireless local area network, there are multiple mobile users that communicate via fixed Access Points (APs), as shown in Figure 1. At a given point in time, a mobile user associates with one of the APs, and all user communications go through this AP. Users can move between different access points.

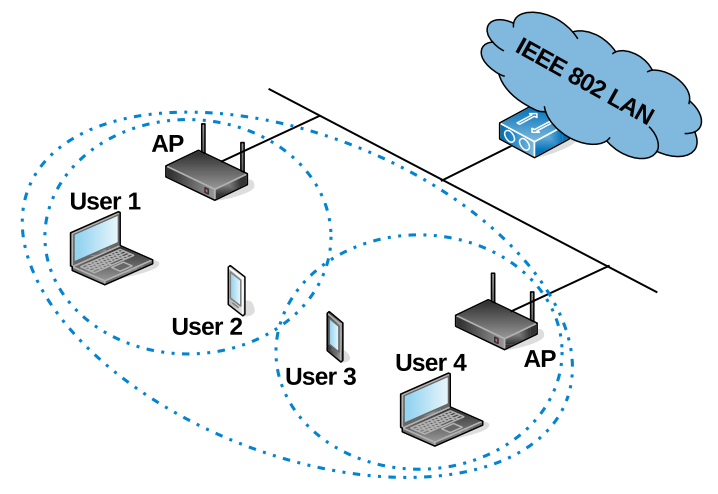

Fig. 1: IEEE WLAN Components

The physical layer transmission parameters, including Modulation and Coding Scheme (MCS) index, channel width, guard interval and MIMO streams, determine the datarate for a given user at a given point in time. An MCS index corresponds to a Modulation type and Coding rate. In IEEE 802.11ac/ax, Quadrature Amplitude Modulation (QAM) is used as the modulation type. The constellation size $(2,4,16,64,256$, 1024) of QAM determines the number of bits that can be encoded per symbol. Higher the number of bits that can be encoded higher is the datarate that can be achieved. However, packet transmission at a higher constellation size is more prone to corruption due to noise. Guard intervals are used to avoid interference between multiple transmissions. A higher datarate can be achieved by using a smaller guard interval, but chances of transmission overlap increases which may lead to packet corruption. The optimal set of parameters will ensure that the target bit error rate is not violated, while at the same time, the user transmits at the best possible data rate.

For instance, in IEEE 802.11ac, the channel width is selected from the set $\{20,40,80,160\} \mathrm{MHz}$; the MCS indices range from 0 (BPSK modulation and coding rate of $1 / 2$ ) to 9 (256-QAM modulation and coding rate of 5/6); the guard interval values are $0.4 \mu \mathrm{s}$ and $0.8 \mu \mathrm{s}$; and the number of MIMO streams varies from 1 to 4 . The corresponding data rates vary from 6.5 Mbps to $6.933 \mathrm{Gbps}$ [14].

\section{B. Related Work}

Prior research in IEEE $802.11 \mathrm{a} / \mathrm{b} / \mathrm{g}$ rate adaptation includes mechanisms such as Adaptive Auto Rate Fallback (AARF) [15], Robust Rate Adaptation Algorithm (RRAA) [16], Loss Differentiating Rate Adaptation (LDRA) [17], Minstrel [18]. As these works were proposed for rate adaptation in IEEE $802.11 \mathrm{a} / \mathrm{b} / \mathrm{g}$, they consider only limited number of datarates and thus would not work well for High-Throughput WLAN (HT-WLAN). Minstrel was later adapted for HT-WLAN (802.11n/ac) in Minstrel-HT [19].

Minstrel-HT is the default rate adaptation algorithm for Linux systems with Atheros wireless chipsets. It maintains a retry chain of rates with highest expected throughput, 2nd highest expected throughput and highest probability of success. $90 \%$ of the times, the algorithm transmits packets using this retry chain. $10 \%$ of the time it uses a different retry chain by sampling a rate from all the remaining rates and replacing the rate with 2 nd highest expected throughput with the sampled rate. Minstrel-HT would work well where the mobile devices are mostly stationary. However, in a mobile environment, Mintrel-HT would not perform well as it does not consider the receiver mobile device's RSSI value.

SampleLite [7] tries to reduce the sample space by identifying the rates suitable for different RSSI ranges for IEEE 802.11 n. It first measures the RSSI value of the mobile device and samples a rate from the corresponding set of rates suitable for that RSSI value. Though SampleLite considers the receiver's RSSI range, it maps each RSSI range to only three datarates. SampleLite might not determine the optimal datarate as the same RSSI range can have different optimal datarate in different environments, thus the mapping to only three datarates may not hold true every time.

Recently, many published works [1]-[4] have used Reinforcement Learning techniques for rate adaptation. All of them use the Multi-armed Bandit (MAB) problem setting to find the optimal datarate. In [1] and [3], the authors argue that the success probabilities for the lower rates are higher as compared to that of the higher rates and then proposes a rate adaptation algorithm which tries to exploit this structure. The authors define the probability of success of a datarate as the summation of the probabilities of occurrence of the channel conditions in which a packet can be successfully transmitted using the datarate. However, this definition does not capture 
the evolution of the success probabilities of the datarates with the variation of the channel conditions. As a result, the rate adaptation algorithm may choose a sub-optimal datarate in a mobile environment.

In [2], the authors propose rate adaptation algorithms for both stationary and non-stationary radio environments. The throughput is modelled as a unimodal function of the datarates, which is exploited in the rate adaptation algorithms. However, the rate adaptation algorithms use all the available datarates to find out the optimal rate. This results in datarates, unsuitable for a particular channel condition, being used and so overall throughput reduces. Additionally, the time taken to find out the optimal rate also is high because of this reason. In [4] also rate adaptation algorithms are proposed for both stationary and non-stationary radio environments. The rate adaptation algorithms only go through a reduced rate set for finding out the optimal rate. However, the rate adaptation algorithms use the same rate set for all channel conditions which may lead to reduced overall throughput as datarates unsuitable for a particular channel condition may be used.

TABLE I: Comparison of different rate adaptation algorithms

\begin{tabular}{ccccc}
\hline Technique & $\begin{array}{c}\mathbf{8 0 2 . 1 1} \\
\text { standard }\end{array}$ & $\begin{array}{c}\text { Reinforcement } \\
\text { Learning based }\end{array}$ & $\begin{array}{c}\text { Channel } \\
\text { condition } \\
\text { feedback }\end{array}$ & $\begin{array}{c}\text { Channel } \\
\text { condition } \\
\text { specific } \\
\text { rate sets }\end{array}$ \\
\hline$[15]-[18]$ & $802.11 \mathrm{a} / \mathrm{b} / \mathrm{g}$ & No & No & No \\
{$[19]$} & $802.11 \mathrm{n} / \mathrm{ac}$ & No & No & No \\
{$[7]$} & $802.11 \mathrm{n}$ & No & Yes & Yes \\
{$[1]$} & $802.11 \mathrm{~g} / \mathrm{n}$ & Yes & No & No \\
{$[2]$} & $802.11 \mathrm{~g} / \mathrm{n}$ & Yes & No & No \\
{$[3]$} & $802.11 \mathrm{~g} / \mathrm{n}$ & Yes & No & No \\
{$[4]$} & $802.11 \mathrm{ac}$ & Yes & No & No \\
$\begin{array}{c}\text { Proposed } \\
\text { algorithm }\end{array}$ & $802.11 \mathrm{ac} / \mathrm{ax}$ & Yes & Yes & Yes \\
\hline
\end{tabular}

Table I presents the comparison of existing works with the proposed rate adaptation algorithm. As the proposed algorithm is based on a Reinforcement Learning method, it optimally explores the sub-optimal rates in order to determine the optimal rate for maximizing the overall throughput. The non-Reinforcement Learning techniques [7], [15]-[19] use some heuristic to find out the optimal solution and thus may achieve reduced overall throughput. The Reinforcement Learning based techniques [1]-[4] do not directly use the information about the channel conditions and also do not use channel condition specific rate sets. This may result in reduced throughput and delayed determination of the optimal rate for a particular channel condition. Some of the algorithms may even fail to find out a optimal rate in a mobile environment.

Moreover, all the rate adaptation algorithms try to find out the optimal rate for each individual links (sender-receiver pair). As a result, the sender tries to find out the optimal rate every time a new receiver comes into the system even if the sender may have already found out the optimal rate for the particular channel condition the new receiver is in. As the proposed rate adaptation algorithm runs only a single instance for all the receivers, it can use the previously learnt information for the new receivers. Thus, it can quickly determine the optimal rates for the different channel conditions.

\section{Contextual Bandit Problem}

In this work, we consider a single Access Point (AP) that multiple mobile users are associated with. This can be extended to multiple-AP scenarios, suitably.

\section{TABLE II: Summary of Notations}

\begin{tabular}{|l|l|}
\hline Notation & Description \\
\hline$x_{t}$ & RSSI range the receiver is in at timestep $t$ \\
$X$ & Set of all RSSI ranges \\
$\mathbb{A}$ & Set of all possible datarates \\
$f: X \mapsto \mathbb{A}$ & Gives the rate set for an RSSI range \\
$A_{t}$ & Datarate set at timestep $t$ \\
$a_{x_{t}}$ & A datarate in the set $A_{t}$ \\
$n_{t}$ & The cardinality of the set $A_{t}$ \\
$a_{x_{t}, i}$ & ith datarate in the set $A_{t}$ \\
$p_{x_{t}}()$. & Probability of success of a rate in RSSI range $x_{t}$ \\
$a_{x_{t}}^{*}$ & Optimal datarate for RSSI range $x_{t}$ at timestep $t$ \\
$\alpha, \beta$ & Parameters of the Beta function as well as Beta distribution \\
$\alpha_{a_{x}}, \beta_{a_{x}}$ & Beta distribution parameters corresponding to rate $a_{x} \in A$, \\
$\hat{\theta}_{x_{t}}$ & where $A=f(x)$ \\
$\hat{a}_{x_{t}}$ & Sampled probability of success for rates at timestep $t$ \\
$\hat{r}_{x_{t}}$ & Datarate selected for RSSI range $x_{t}$ at timestep $t$ \\
$\delta$ & Reward obtained for selected datarate $\hat{a}_{x_{t}}$ \\
$A_{t}^{\prime}$ & Threshold probability \\
$R S S I_{j}$ & Set of rates with sampled probability of success $\hat{\theta}_{x_{t}}$ greater \\
$t_{j}$ & than $\delta$ \\
$R S S I_{j}^{c u r r}\left(t_{j}\right)$ & Average RSSI value of mobile device $j$ \\
$\gamma$ & Timestep at which the RSSI value of mobile device $j$ is \\
& measured \\
& Measured RSSI at timestep $t_{j}$ for mobile $j$ \\
& The degree of weighting decrease of average RSSI value \\
\hline
\end{tabular}

We have considered the Contextual Bandit setting for the rate adaptation in a mobile environment. All the notations used to model rate adaptation in a mobile environment as a Contextual Bandit problem are described in Table II. Every packet transmission is considered as a timestep $(t)$. The RSSI range, suitable for packet transmission, is divided into subranges. Let $X$ be the set of all the sub-ranges. Before a packet is sent, the sub-range in which the RSSI of the receiver mobile device lies is identified $\left(x_{t} \in X\right)$ and that is considered as the context for the timestep $t . f\left(x_{t}\right)$ defined as $f: X \mapsto \mathbb{A}$, where $\mathbb{A}=$ \{all possible rates $\}$, gives the rate set $A_{t} \subseteq \mathbb{A}$ for context $x_{t}$. Let $n_{t}$ denote the cardinality of the set $A_{t}$, then we can define $A_{t}$ as $\left\{a_{x_{t}, 1}, \ldots, a_{x_{t}, n_{t}}\right\}$. A rate $\left(a_{x_{t}} \in A_{t}\right)$ is selected and the packet is transmitted at that rate. For every successful packet transmission a reward of 1 is received and if the transmission fails a reward of 0 is received. This is a special type of MAB problem called Bernoulli Bandit problem. Let the probability of success for rate $a \in A_{t}$ be denoted as $p_{x_{t}}(a)$. This means that at timestep $\mathrm{t}$ if the receiver mobile device's RSSI lies in $x_{t}$ and if rate $a$ is used to transmit a packet, then the probability of getting 1 as reward is $p_{x_{t}}(a)$, and the probability of getting 0 as reward is $1-p_{x_{t}}(a)$. However, the actual probability distribution of $p_{x_{t}}(a)$ is unknown. Let $\operatorname{Reward}(T)$ denote the expected total reward after timestep $T$. Mathematically,

$$
\operatorname{Reward}(T)=\mathbb{E}\left[\sum_{t=1}^{T} p_{x_{t}}\left(a_{x_{t}}\right)\right]
$$

It is to be noted that to maximize the total reward, at each timestep the rate with highest probability of success should be chosen. However, choosing this rate may lead to sub-optimal overall throughput. For this reason, rather than maximizing the expected total reward $\operatorname{Reward}(T)$, the expected total 


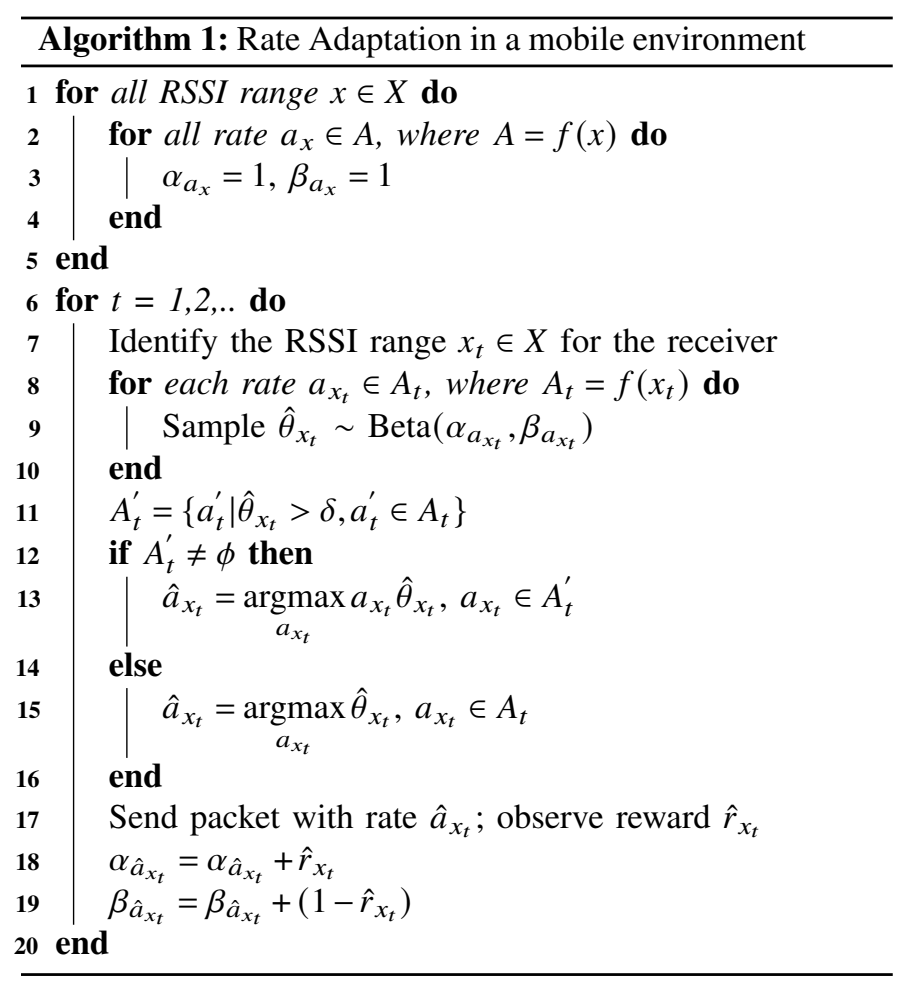

throughput should be maximized. Let $\operatorname{Thr}(T)$ denote the expected total throughput after $T$ timesteps. Mathematically,

$$
\operatorname{Thr}(T)=\mathbb{E}\left[\sum_{t=1}^{T} a_{x_{t}} p_{x_{t}}\left(a_{x_{t}}\right)\right]
$$

The goal of the rate adaptation algorithm can also be expressed in terms of minimizing the expected total regret. Regret is defined as the amount of throughput lost for not choosing the optimal rate. Mathematically, the expected total regret after $\mathrm{T}$ timesteps is:

$$
\operatorname{Regret}(T)=\mathbb{E}\left[\sum_{t=1}^{T}\left[a_{x_{t}}^{*} p_{x_{t}}\left(a_{x_{t}}^{*}\right)-a_{x_{t}} p_{x_{t}}\left(a_{x_{t}}\right)\right]\right]
$$

where $a_{x_{t}}^{*}$ is the optimal rate for at timestep $t$ and its expected throughput at timestep $t$ is given by $a_{x_{t}}^{*} p_{x_{t}}\left(a_{x_{t}}^{*}\right)$. The efficiency of the rate adaptation algorithm can also be expressed by finding the regret bounds.

\section{RATE AdAPtATion Algorithm In A MOBile ENVIRONMENT}

This section presents the proposed rate adaptation algorithm, based on the Context Bandit problem.

\section{A. Proposed Algorithm}

The objective of the algorithm is to maximize the overall throughput by determining the datarates with highest expected throughput for different RSSI ranges. In order to maximize the overall throughput, the rate adaptation algorithm should try to estimate the probability distribution $p_{x_{t}}($.$) for context$ $x_{t}$. To estimate the probability distribution for finding out the optimal rate, the rate adaptation algorithm should try out more rates (exploration). However, to maximize the total throughput, the algorithm should choose the rate which currently has the highest expected throughput (exploitation). There are many methods which try to address this exploration-exploitation dilemma, namely $\epsilon$-Greedy [5], Upper Confidence Bound (UCB) [5], Thompson Sampling [20], etc. In this paper, we use the Thompson Sampling method since it was generally observed to perform better than other existing methods [21].

Thompson Sampling estimates the probability distribution of $p_{x_{t}}($.$) by taking an initial guess (prior) and then updating$ the estimated distribution (posterior) every timestep after observing the reward. For each action, a sample is drawn from the corresponding estimated probability distribution and the action with highest expected reward is chosen based on the samples. In our proposed method, we will be choosing the rate with highest expected throughput. The reward is observed for the chosen action and the corresponding estimated probability distribution is updated using Bayes' rule. For the Bernoulli Bandit problem, the Beta distribution is usually used as a prior [22]. The probability density function for Beta distribution with parameters $\alpha$ and $\beta$ is given by:

$$
\mathcal{P}(\lambda ; \alpha, \beta)=\frac{\lambda^{\alpha-1}(1-\lambda)^{\beta-1}}{B(\alpha, \beta)}, \text { where } \lambda \in[0,1]
$$

Here, $B(\alpha, \beta)$ is the Beta function with parameters $\alpha$ and $\beta$. The Beta function is given by:

$$
B(\alpha, \beta)=\int_{0}^{1} u^{\alpha-1}(1-u)^{\beta-1} d u
$$

For all the actions $(\alpha, \beta)$ are initialized to $(1,1)$. For the chosen action, the corresponding posterior will also follow the Beta distribution with parameters:

$$
(\alpha, \beta)= \begin{cases}(\alpha+1, \beta), & \text { if the reward is } 1 \\ (\alpha, \beta+1), & \text { if the reward is } 0\end{cases}
$$

In the rate adaptation setting, for each rate $a_{x_{t}} \in A_{t}$ let the Beta distribution parameters be denoted by $\left(\alpha_{a_{x_{t}}}, \beta_{a_{x_{t}}}\right)$.

Algorithm 1 presents the proposed algorithm for rate adaptation in a mobile environment. For each RSSI range $x \in X$, the $\left(\alpha_{a_{x}}, \beta_{a_{x}}\right)$ values are initialized to $(1,1)$ for all the rates $a_{x} \in A$, where $A=f(x)$. Let there be $N$ receiver mobile

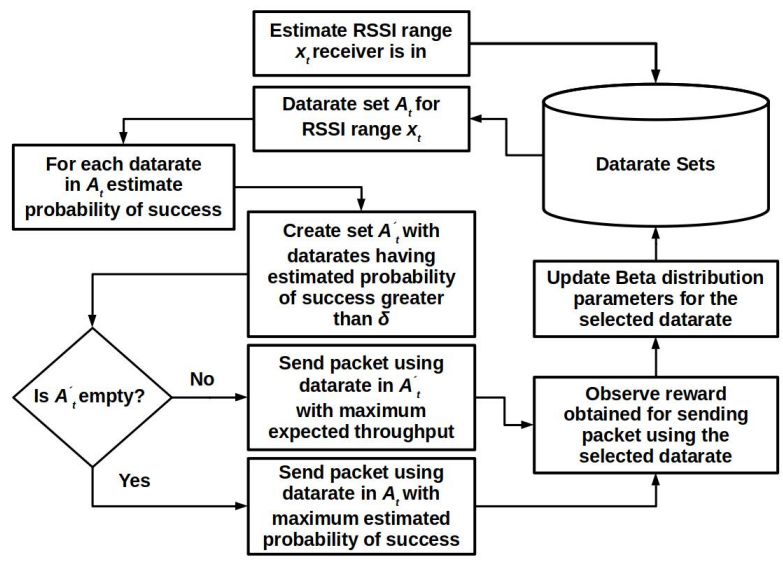

Fig. 2: Flow diagram of the Rate Adaptation Algorithm 
devices. For each mobile device $j \in\{1, . ., N\}$, an average RSSI value $\left(R S S I_{j}\right)$ is maintained using the Exponential Weighted Moving Average (EWMA). Let $t_{j}$ denote the timestep at which the RSSI value of mobile device $j$ is measured by the sender. Then, the average RSSI $\left(R S S I_{j}\right)$ for mobile device $j$ for future packet transmission is estimated using the following equation:

$$
R S S I_{j}=\gamma * R S S I_{j}^{\text {curr }}\left(t_{j}\right)+(1-\gamma) * R S S I_{j}
$$

The measured RSSI at timestep $t_{j}$ for mobile $j$ is represented by $R S S I_{j}^{\text {curr }}\left(t_{j}\right) . R S S I_{j}^{\text {curr }}\left(t_{j}\right)$ is the RSSI of the ACK frame sent by mobile $j$ on successfully receiving the packet sent to it at timestep $t_{j} \cdot \gamma \in[0,1]$ represents the degree of weighting decrease. Every time a packet is sent to a mobile device $j$ (timestep $t$ ), the corresponding $R S S I_{j}$ is used to identify the RSSI range $\left(x_{t} \in X\right)$ for the receiver mobile device. The subsequent steps of the proposed algorithm are presented in figure 2. For each rate $a_{x_{t}} \in A_{t}$, where $A_{t}=f\left(x_{t}\right)$, a sample $\hat{\theta}_{x_{t}}$ is drawn from the Beta distribution with parameters $\left(\alpha_{a_{x_{t}}}, \beta_{a_{x_{t}}}\right)$. These samples are used as the estimated expected probabilities of successful packet transmission at timestep $t$ for the corresponding rates. The rate set $A_{t}^{\prime}$ is obtained by considering only those rates which have the estimated probability of success $\hat{\theta}_{x_{t}}$ greater than a threshold $\delta$. If the set $A_{t}^{\prime}$ is not empty, $\mathrm{t}$ the rate $\hat{a}_{x_{t}}$ with the highest expected throughput $\hat{a}_{x_{t}} \hat{\theta}_{x_{t}}$ is chosen from the set $A_{t}^{\prime}$. If the set $A_{t}^{\prime}$ is empty, the rate $\hat{a}_{x_{t}}$ with the highest expected probability of success $\hat{\theta}_{x_{t}}$ is chosen from the set $A_{t}$. Since, all the datarates have an estimated probability of success less than the threshold value, choosing the datarate with highest estimated throughput may result in a packet loss. Thus, choosing the datarate with highest estimated probability of success can avoid the packet loss. The packet is sent using the rate $\hat{a}_{x_{t}}$ and the reward $\hat{r}_{x_{t}}$ is observed. If the packet transmission is successful, $\alpha_{\hat{a}_{x_{t}}}$ will be incremented by 1 ; otherwise, $\beta_{\hat{a}_{x_{t}}}$ will be incremented by 1.

\section{B. Datarate sets for different channel conditions}

As shown in [6], some configurations work well for IEEE 802.11ac across a range of RSSI values; however, for some other RSSI ranges, using the same configurations may lead to high packet loss. For example, when the RSSI value is greater than $-53 \mathrm{dBm}$, high throughput can be achieved with channel width either $160 \mathrm{MHz}$ or $80 \mathrm{MHz}$ and Modulation and Coding Scheme (MCS) values 8 and 9. However, the same set of configurations may lead to high packet loss for RSSI values less than $-75 \mathrm{dBm}$. Thus, for RSSI values greater than $-53 \mathrm{dBm}$, the rate set should comprise of the rates corresponding to the configurations mentioned above. Based on the type of datarate sets chosen for the RSSI ranges, we propose two versions of the ContRA algorithm: General ContRA (GeContRA) algorithm and Best Effort ContRA (BEContRA) algorithm.

1) General ContRA (GeContRA) Algorithm: In GeContRA algorithm, all the datarates available in IEEE 802.11ac/ax are considered. The datarates are divided into different sets and assigned to the suitable RSSI ranges.

Table III shows the GeContRA algorithm rate sets for different RSSI ranges for IEEE 802.11ac. For each RSSI range, the rates are decided by the combination of the MCS values, the channel widths, the guard intervals and the number of MIMO streams assigned to that RSSI range. Both the guard intervals $(0.4 \mu \mathrm{s}$ and $0.8 \mu \mathrm{s})$ and all the MIMO streams $(1-4)$ of IEEE 802.11ac are considered for all the RSSI ranges. A configuration is chosen for a mobile user only if the configuration is supported by it. The channel widths and the MCS values suitable for different RSSI ranges for IEEE $802.11 \mathrm{ac}$ are identified from the study in [6].

However, for the new IEEE 802.11ax standard, such kind of study is currently unavailable. The number of rates available in IEEE 802.11ax has increased from that of IEEE 802.11ac due to the introduction of two additional MCS values (10 and 11) and the replacement of the guard intervals of $0.4 \mu \mathrm{s}$ and $0.8 \mu \mathrm{s}$ with $0.8 \mu \mathrm{s}, 1.6 \mu \mathrm{s}$ and $3.2 \mu \mathrm{s}$. Table IV shows the GeContRA algorithm rate sets for different RSSI ranges for

TABLE III: General ContRA algorithm rate sets for different RSSI ranges for IEEE 802.11ac

\begin{tabular}{|c|c|c|c|}
\hline RSSI range & Channel width \& MCS & Guard interval & $\begin{array}{l}\text { MIMO } \\
\text { streams }\end{array}$ \\
\hline$<-75 \mathrm{dBm}$ & $\begin{array}{l}20 M H z \&\{0-9\}, \\
40 M H z \&\{0-9\}, \\
80 M H z \&\{0-9\}\end{array}$ & $0.4 \mu \mathrm{s} \& 0.8 \mu \mathrm{s}$ & $1-4$ \\
\hline $\begin{array}{c}<-70 \mathrm{dBm} \& \\
\geq-75 \mathrm{dBm}\end{array}$ & $\begin{array}{l}20 M H z \&\{3-9\} \\
40 M H z \&\{2-9\} \\
80 M H z \&\{1-9\} \\
160 M H z \&\{0-9\}\end{array}$ & $0.4 \mu \mathrm{s} \& 0.8 \mu \mathrm{s}$ & $1-4$ \\
\hline $\begin{array}{c}<-66 \mathrm{dBm} \& \\
\geq-70 \mathrm{dBm}\end{array}$ & $\begin{array}{l}20 M H z \&\{4-9\}, \\
40 M H z \&\{4-9\}, \\
80 M H z \&\{3-9\}, \\
160 M H z \&\{1-9\}\end{array}$ & $0.4 \mu \mathrm{s} \& 0.8 \mu \mathrm{s}$ & $1-4$ \\
\hline $\begin{array}{c}<-63 \mathrm{dBm} \& \\
\geq-66 \mathrm{dBm}\end{array}$ & $\begin{array}{l}20 M H z \&\{5-9\} \\
80 M H z \&\{4-9\} \\
160 M H z \&\{3-9\}\end{array}$ & $0.4 \mu \mathrm{s} \& 0.8 \mu \mathrm{s}$ & $1-4$ \\
\hline $\begin{array}{c}<-60 \mathrm{dBm} \& \\
\geq-63 \mathrm{dBm}\end{array}$ & $\begin{array}{l}40 M H z \&\{5-9\} \\
160 M H z \&\{4-9\}\end{array}$ & $0.4 \mu \mathrm{s} \& 0.8 \mu \mathrm{s}$ & $1-4$ \\
\hline $\begin{array}{c}<-53 \mathrm{dBm} \& \\
\geq-60 \mathrm{dBm}\end{array}$ & $\begin{array}{c}20 M H z \&\{8,9\} \\
40 M H z \&\{8,9\} \\
80 M H z \&\{5-9\} \\
160 M H z \&\{5-9\}\end{array}$ & $0.4 \mu \mathrm{s} \& 0.8 \mu \mathrm{s}$ & $1-4$ \\
\hline$\geq-53 \mathrm{dBm}$ & $\begin{array}{l}80 M \mathrm{MH} \&\{8,9\} \\
160 \mathrm{MHz} \&\{8,9\}\end{array}$ & $0.4 \mu \mathrm{s} \& 0.8 \mu \mathrm{s}$ & $1-4$ \\
\hline
\end{tabular}

TABLE IV: General ContRA algorithm rate sets for different RSSI ranges for IEEE 802.11ax

\begin{tabular}{|c|c|c|c|}
\hline RSSI range & Channel width \& MCS & Guard interval & $\begin{array}{l}\text { MIMO } \\
\text { streams }\end{array}$ \\
\hline$<-75 \mathrm{dBm}$ & $\begin{array}{l}20 M H z \&\{0-11\} \\
40 M H z \&\{0-11\} \\
80 M H z \&\{0-11\}\end{array}$ & $\begin{array}{l}0.8 \mu \mathrm{s}, 1.6 \mu \mathrm{s} \text { and } \\
3.2 \mu \mathrm{s}\end{array}$ & $1-4$ \\
\hline $\begin{array}{l}<-70 \mathrm{dBm} \& \\
\geq-75 \mathrm{dBm}\end{array}$ & $\begin{array}{l}20 M H z \&\{3-11\} \\
40 M H z \&\{2-11\}, \\
80 M H z \&\{1-11\}, \\
160 M H z \&\{0-11\}\end{array}$ & $\begin{array}{l}0.8 \mu \mathrm{s}, 1.6 \mu \mathrm{s} \text { and } \\
3.2 \mu \mathrm{s}\end{array}$ & $1-4$ \\
\hline $\begin{array}{l}<-66 \mathrm{dBm} \& \\
\geq-70 \mathrm{dBm}\end{array}$ & $\begin{array}{l}20 M H z \&\{4-11\} \\
40 M H z \&\{4-11\}, \\
80 M H z \&\{3-11\}, \\
160 M H z \&\{1-11\}\end{array}$ & $\begin{array}{l}0.8 \mu \mathrm{s}, 1.6 \mu \mathrm{s} \text { and } \\
3.2 \mu \mathrm{s}\end{array}$ & $1-4$ \\
\hline $\begin{array}{c}<-63 \mathrm{dBm} \& \\
\geq-66 \mathrm{dBm}\end{array}$ & $\begin{array}{l}20 M H z \&\{5-11\} \\
80 M H z \&\{4-11\} \\
160 M H z \&\{3-11\}\end{array}$ & $\begin{array}{l}0.8 \mu \mathrm{s}, 1.6 \mu \mathrm{s} \text { and } \\
3.2 \mu \mathrm{s}\end{array}$ & $1-4$ \\
\hline $\begin{array}{c}<-60 \mathrm{dBm} \& \\
\geq-63 \mathrm{dBm} \\
\end{array}$ & $\begin{array}{l}40 M H z \&\{5-11\} \\
160 M H z \&\{4-11\}\end{array}$ & $\begin{array}{l}0.8 \mu \mathrm{s}, 1.6 \mu \mathrm{s} \text { and } \\
3.2 \mu \mathrm{s}\end{array}$ & $1-4$ \\
\hline $\begin{array}{c}<-53 \mathrm{dBm} \& \\
\geq-60 \mathrm{dBm}\end{array}$ & $\begin{array}{l}20 M H z \&\{8-11\} \\
40 M H z \&\{8-11\}, \\
80 M H z \&\{5-11\}, \\
160 M H z \&\{5-11\}\end{array}$ & $\begin{array}{l}0.8 \mu \mathrm{s}, 1.6 \mu \mathrm{s} \text { and } \\
3.2 \mu \mathrm{s}\end{array}$ & $1-4$ \\
\hline$\geq-53 \mathrm{dBm}$ & $\begin{array}{l}20 M H z \&\{10,11\} \\
40 M H z \&\{10,11\} \\
80 M H z \&\{8-11\} \\
160 M H z \&\{8-11\}\end{array}$ & $\begin{array}{l}0.8 \mu \mathrm{s}, 1.6 \mu \mathrm{s} \text { and } \\
3.2 \mu \mathrm{s}\end{array}$ & $1-4$ \\
\hline
\end{tabular}


IEEE 802.11ax. All the MIMO streams $(1-4)$ are also considered for IEEE 802.11ax. For this case too, a configuration is chosen for a mobile user only if the configuration is supported by it. In the case of IEEE $802.11 \mathrm{ax}$, the GeContRA algorithm rate set for each RSSI range is determined by keeping the channel width configurations same and replacing the old guard intervals with the new ones. The two new MCS values for all the channel widths are added to the configurations of all the RSSI ranges.

The selection of different rate sets for different RSSI ranges, makes the sizes of these sets much smaller as compared to the size of the set of all possible rates. As a result, the time for the proposed algorithm to determine an optimal rate will be expedited. Additionally, as the channel conditions improve the lower datarates are excluded from the rate sets. This ensures that for better channel conditions only the higher datarates are used. While trying to find out the optimal rate, the algorithm will not try rates which may lead to very low throughput or very high packet loss. It will only try those rates which are suitable for that RSSI range. Thus, the overall throughput will be maximized.

For example, if a receiver has an RSSI value above $-53 \mathrm{dBm}$ then the sender will have to choose a datarate from the rate set associated with that RSSI range. Many lower datarates are not included in this associated rate set as they will give sub-optimal throughput value. If the receiver's RSSI value decreases and lies between $-53 \mathrm{dBm}$ and $-60 \mathrm{dBm}$ then the receiver will choose from a different rate set. Though, this rate set includes the datarates used for the RSSI range above $-53 \mathrm{dBm}$, their probability of success will be lower in this RSSI range. Thus, for each datarate separate $\alpha$ and $\beta$ values are maintained for different RSSI ranges. This captures the fact

TABLE V: Best Effort ContRA algorithm rate sets for different RSSI ranges for IEEE 802.11ac

\begin{tabular}{|c|c|c|c|}
\hline RSSI range & Channel width \& MCS & Guard interval & $\begin{array}{l}\text { MIMO } \\
\text { streams }\end{array}$ \\
\hline$<-75 \mathrm{dBm}$ & $\begin{array}{c}20 M H z \&\{0\}, \\
40 M H z \&\{0\}, \\
80 M H z \&\{0\}, \\
160 M H z \&\{0-9\}\end{array}$ & $0.4 \mu \mathrm{s} \& 0.8 \mu \mathrm{s}$ & $1-4$ \\
\hline $\begin{array}{c}<-70 \mathrm{dBm} \& \\
\geq-75 \mathrm{dBm}\end{array}$ & $160 M H z \&\{1-9\}$ & $0.4 \mu \mathrm{s} \& 0.8 \mu \mathrm{s}$ & $1-4$ \\
\hline $\begin{array}{c}<-60 \mathrm{dBm} \mathrm{\&} \\
\geq-70 \mathrm{dBm}\end{array}$ & $160 M H z \&\{2-9\}$ & $0.4 \mu \mathrm{s} \& 0.8 \mu \mathrm{s}$ & $1-4$ \\
\hline $\begin{array}{c}<-55 \mathrm{dBm} \& \\
\geq-60 \mathrm{dBm}\end{array}$ & $160 M H z \&\{5-9\}$ & $0.4 \mu \mathrm{s} \& 0.8 \mu \mathrm{s}$ & $1-4$ \\
\hline$\geq-55 \mathrm{dBm}$ & $160 \mathrm{MHz} \&\{8,9\}$ & $0.4 \mu \mathrm{s} \& 0.8 \mu \mathrm{s}$ & $1-4$ \\
\hline
\end{tabular}

TABLE VI: Best Effort ContRA algorithm rate sets for different RSSI ranges for IEEE 802.11ax

\begin{tabular}{|c|c|c|c|}
\hline RSSI range & Channel width \& MCS & Guard interval & $\begin{array}{l}\text { MIMO } \\
\text { streams }\end{array}$ \\
\hline$<-75 \mathrm{dBm}$ & $\begin{array}{c}20 M H z \&\{0\}, \\
40 M H z \&\{0\}, \\
80 M H z \&\{0\}, \\
160 M H z \&\{0-11\}\end{array}$ & $\begin{array}{l}0.8 \mu \mathrm{s}, 1.6 \mu \mathrm{s} \text { and } \\
3.2 \mu \mathrm{s}\end{array}$ & $1-4$ \\
\hline $\begin{array}{c}<-70 \mathrm{dBm} \& \\
\geq-75 \mathrm{dBm}\end{array}$ & $160 M H z \&\{1-11\}$ & $\begin{array}{l}0.8 \mu \mathrm{s}, 1.6 \mu \mathrm{s} \text { and } \\
3.2 \mu \mathrm{s}\end{array}$ & $1-4$ \\
\hline $\begin{array}{c}<-60 \mathrm{dBm} \& \\
\geq-70 \mathrm{dBm}\end{array}$ & $160 M H z \&\{2-11\}$ & $\begin{array}{l}0.8 \mu \mathrm{s}, 1.6 \mu \mathrm{s} \text { and } \\
3.2 \mu \mathrm{s}\end{array}$ & $1-4$ \\
\hline $\begin{array}{c}<-55 \mathrm{dBm} \& \\
\geq-60 \mathrm{dBm}\end{array}$ & $160 M H z \&\{5-11\}$ & $\begin{array}{l}0.8 \mu \mathrm{s}, 1.6 \mu \mathrm{s} \text { and } \\
3.2 \mu \mathrm{s}\end{array}$ & $1-4$ \\
\hline$\geq-55 \mathrm{dBm}$ & $160 M H z \&\{8-11\}$ & $\begin{array}{l}0.8 \mu \mathrm{s}, 1.6 \mu \mathrm{s} \text { and } \\
3.2 \mu \mathrm{s}\end{array}$ & $1-4$ \\
\hline
\end{tabular}

that same datarate can give maximum throughput in one RSSI range and in another RSSI range it may not be so. The ContRA algorithm tries to determine the datarate for each RSSI range which will give the maximum throughput for that range, thus maximizing the overall throughput.

2) Best Effort ContRA (BEContRA) Algorithm: In the BEContRA algorithm, only the highest datarates for certain channel conditions are considered. These datarates are also identified from the study in [6]. The datarates are divided into different sets and assigned to the suitable RSSI ranges.

Tables V and VI show the BEContRA algorithm rate sets for different RSSI ranges for IEEE 802.11ac and IEEE 802.11ax respectively. The selection of only the highest datarates for certain channel conditions, reduces the rate sets even further. Thus, exploration of the different rates for a particular RSSI range will not take much time and it will be easier to find out the datarate which maximizes the throughput for that RSSI range. Additionally, as many lower datarates are not included, exploration of lower sub-optimal datarates is also avoided. Thus, the optimal datarate for a particular RSSI range can be determined quickly. Similar to GeContRA, in BEContRA also a configuration is chosen for a mobile user if the configuration is supported by it.

For example, the size of the rate set associated with the RSSI range above $-55 \mathrm{dBm}$ in BEContRA is much smaller than that of the rate set for RSSI range above $-53 \mathrm{dBm}$ in GeContRA. Additionally, only the higher datarates are considered in BEContRA. As a result, BEContRA avoids the lower datarates and tries to maximize the overall throughput.

\section{PERformance EVAluation}

The proposed ContRA algorithms are implemented in the ns3 network simulator (version 3.30) [13]. The performance of the proposed ContRA algorithms is evaluated in three mobility scenarios: (a) A single mobile device moving away from the AP, (b) A single mobile device moving towards the AP, and (c) Multiple mobile devices moving in random directions within the coverage area of the AP. In all the scenarios, the mobile devices move with constant speed. Additionally, the scenarios are run for both IEEE 802.11ac and IEEE 802.11ax. The EWMA parameter $\gamma$ is assigned a value of 0.9 and the probability threshold $\delta$ is assigned a value of 0.5 .

The performance results of the proposed ContRA algorithm (both the General and the Best Effort versions) are compared with that of the Rate Adaptation with General Thompson Sampling (RAGTS) and Rate Adaptation with Sliding-Window Thompson Sampling (RASWTS) algorithms which are proposed in [4]. Both RAGTS and RASWTS use multi-armed bandit with a reduced rate set for rate adaptation. RAGTS uses general Thompson Sampling method for stationary scenarios. RASWTS uses packet delivery information of only the last $160 \mathrm{~ms}$ for Thompson Sampling method. The sliding window of $160 \mathrm{~ms}$ helps in adapting to the mobile environment.

The main metric considered for performance analysis is application throughput. For the single mobile device simulation scenarios, to show the overall performance of the rate adaptation algorithms, average throughput is used, whereas 


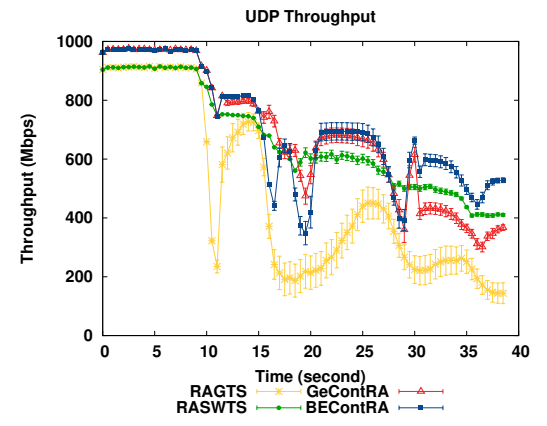

(a) Instantaneous UDP Throughput

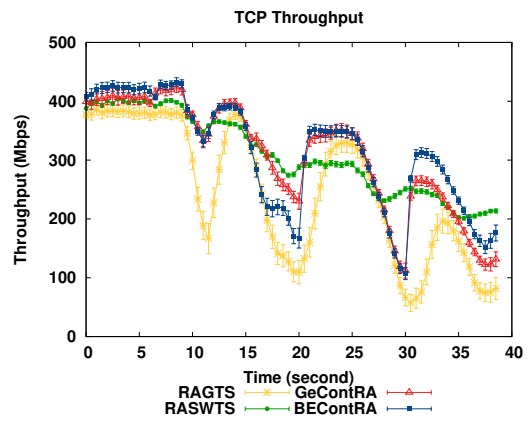

(b) Instantaneous TCP Throughput

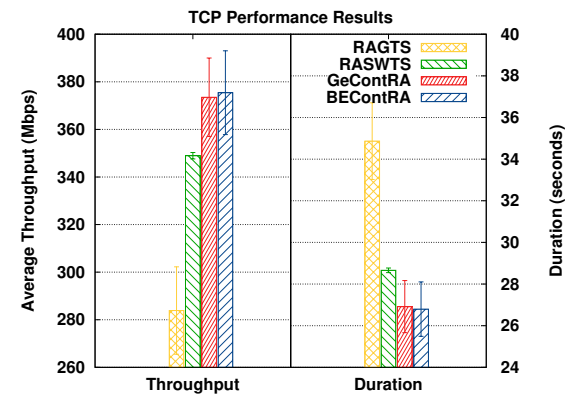

(c) TCP Performance Results

Fig. 3: Mobile device moving away from the AP with constant velocity in IEEE 802.11ac.

instantaneous throughput is used to show immediate effect of the different datarates chosen by the algorithms in different channel conditions. For the multiple mobile device simulation scenario, normalized throughput (per-user average throughput divided by per-user traffic rate), packet delivery percentage (percentage of packets delivered successfully) and packet delay are used for the performance evaluation. All values are plotted with $95 \%$ confidence interval.

The average time taken to determine a datarate for each timestep is approximately $1.2 \mathrm{~ms}$ for GeContRA in both 802.11ac and 802.11ax, 0.9ms for BEContRA in 802.11ac and $1.5 \mathrm{~ms}$ for BEContRA in 802.11ax. These values depend on the system configuration and the load on the system. The system used for the simulations has Intel ${ }^{\mathbb{R}} \mathrm{Xeon}^{\mathrm{B}}$ Processor of the E5 $\mathrm{v} 4$ family, with a frequency of $2.10 \mathrm{GHz}$, and $128 \mathrm{GiB}$ memory. The computation time can be reduced with suitable hardware and software optimization when implemented in ASICs on routers.

\section{A. Single mobile device in IEEE 802.11ac}

This section presents the performance evaluation results for rate adaptation algorithms in IEEE 802.11ac. UDP and TCP applications are setup on the mobile device. The TCP flavour used in the simulations limits the maximum traffic rate within $450 \mathrm{Mbps}$. As a result, for the case of TCP application, all the RA algorithms are limited by a maximum throughput of 450 Mbps.

1) Mobile device moving away from the AP: Figures $3 a$ and $3 b$ present the instantaneous UDP and TCP throughput respectively and table VII presents the overall average throughput in IEEE 802.11ac when a mobile device moves away from the AP with constant velocity. A constant bit-rate traffic of 1 Gbps is sent to the mobile device for both UDP and TCP applications. As the mobile device moves away from the AP,

TABLE VII: Overall Average Throughput for mobile device moving away from the AP with constant velocity.

\begin{tabular}{ccc}
\hline Algorithm & UDP (Mbps) & TCP (Mbps) \\
\hline RAGTS & 480.20 & 246.42 \\
RASWTS & 661.90 & 307.50 \\
GeContRA & 678.90 & 307.23 \\
BEContRA & 704.61 & 312.04 \\
\hline
\end{tabular}

the channel condition continuously deteriorates. Since the RA algorithms attempt to determine the optimal datarate for the varying channel conditions by exploring different datarates, the throughput keeps fluctuating. In the case of UDP application, both the versions of the ContRA algorithm outperform the RAGTS algorithm. The overall average throughput of RAGTS algorithm in the case UDP application is $480.20 \mathrm{Mbps}$ as compared to $678.90 \mathrm{Mbps}$ of GeContRA and 704.61 Mbps of BEContRA. As RAGTS does not take into consideration the current channel condition, it is not able to adapt quickly to the changing channel condition. RASWTS tries to adapt to the changing channel condition by considering only the packet delivery information of the packets sent in the last $160 \mathrm{~ms}$. Though, RASWTS performs better than the proposed ContRA algorithms at certain times, the proposed ContRA algorithms outperforms it for majority of the simulation duration. The overall average throughput achieved by RASWTS in the case of UDP applications is 661.90 Mbps. In the case of TCP application, the overall throughput is limited to a maximum of of $450 \mathrm{Mbps}$. Thus, for all the RA algorithms the maximum achievable throughput is $450 \mathrm{Mbps}$. In this case also the proposed ContRA algorithms outperform RAGTS as it is not able to quickly adapt to the changing channel conditions. The overall average throughput of RAGTS algorithm in the case TCP application is $246.42 \mathrm{Mbps}$ as compared to 307.23 Mbps of GeContRA and 312.04 Mbps of BEContRA. The overall throughput achieved by RASWTS is $307.50 \mathrm{Mbps}$ which is slightly better than the average throughput achieved by GeContRA (307.23 Mbps). Though the performance of GeConTRA is almost identical to BEContRA, in the last 5 seconds of the experiment, GeContRA is not able to achieve higher throughput. For both the ContRA algorithms, the size of the datarate set increases as the channel condition deteriorates. However, the datarate set for the BEContRA algorithm is much smaller as compared to that of the GeContRA algorithm for the RSSI range below $-75 \mathrm{dBm}$. As a result, throughput achieved by GeContRA is a little less in this RSSI range. At certain times RASWTS performs better than the BEContRA algorithm, but the BEContRA algorithm perform better for most of the simulation duration. The ContRA algorithms perform better than both the other algorithms because it estimates the RSSI range the recipient is in and then chooses 


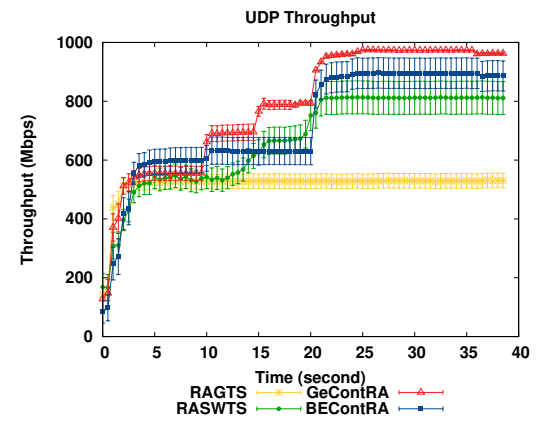

(a) Instantaneous UDP Throughput

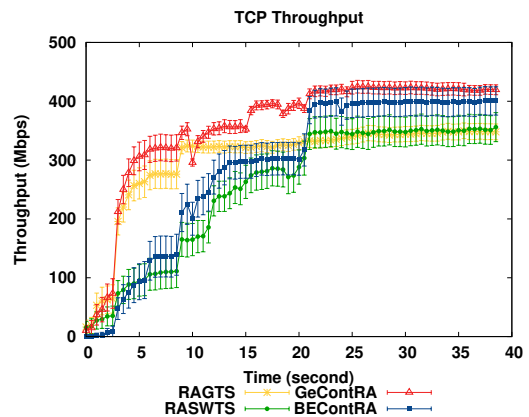

(b) Instantaneous TCP Throughput

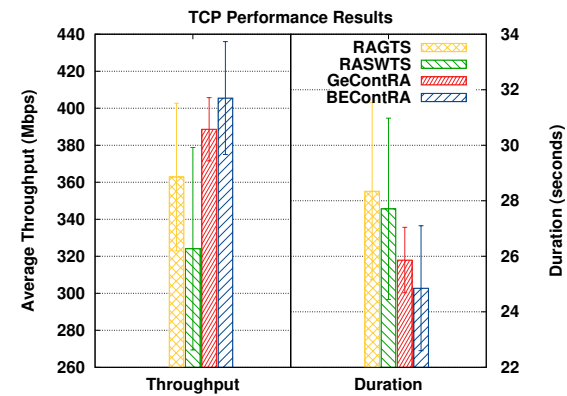

(c) TCP Performance Results

Fig. 4: Mobile device moving towards the AP with constant velocity in IEEE 802.11ac.

from the suitable datarate set. Thus, the overall performance of the ContRA algorithms is better than both the RAGTS and RASWTS algorithms.

Figure $3 c$ presents the average throughput and average duration of a TCP application in IEEE 802.11ac when a mobile device is moving away from the AP. A total of $1.25 \times 10^{9}$ bytes is sent from the sender TCP application layer to the mobile device at one go. The convergence time of the RA algorithms depends on the initial exploration of the datarates and thus also has an effect on the average throughput and duration. Moreover, as the mobile device is moving, the channel conditions keep on changing and with it the optimal datarate. This brings in additional variability as the rate adaptation algorithm has to explore the datarates again for estimating the new optimal datarate. As a result, the confidence intervals are a little bit high as the choices of datarates during the initial exploration for each channel condition affects the overall performance. RAGTS takes the maximum time of 34.9 seconds, followed by RASWTS with 28.7 seconds. GeContRA and BEContRA takes a total 26.9 and 26.8 seconds respectively. Thus, GeContRA and BEContRA provide an improvement of $6.3 \%$ and $6.6 \%$ respectively over RASWTS and an improvement of $22.9 \%$ and $23.2 \%$ respectively over RAGTS in the overall duration. Similarly, GeContRA and BEContRA are able to achieve better throughput of 373.5 Mbps and 375.4 Mbps respectively as compared to 283.9 Mbps and 348.9 Mbps achieved by RAGTS and RASWTS respectively.

2) Mobile device moving towards the AP: Figures 4a and $4 \mathrm{~b}$ present the instantaneous UDP and TCP throughput respectively. Table VIII presents the overall average throughput in IEEE 802.11ac when a mobile device moves towards the AP with constant velocity. A constant bit-rate traffic of 1 Gbps is sent to the mobile device for both UDP and

TABLE VIII: Overall Average Throughput for mobile device moving towards the AP with constant velocity.

\begin{tabular}{ccc}
\hline Algorithm & UDP (Mbps) & TCP (Mbps) \\
\hline RAGTS & 515.99 & 301.83 \\
RASWTS & 670.55 & 254.46 \\
GeContRA & 785.89 & 356.77 \\
BEContRA & 710.92 & 287.54 \\
\hline
\end{tabular}

TCP applications. As the mobile device moves towards the AP, the channel condition continuously improves. As the RA algorithms try to determine the optimal datarate for the varying channel conditions by exploring different datarates, the throughput keeps fluctuating. In the UDP application case, both the versions of the ContRA algorithm mostly outperform the RAGTS algorithm. The overall throughput achieved by RAGTS in the case of UDP applications is 515.99 Mbps as compared to $785.89 \mathrm{Mbps}$ achieved by GeContRA and 710.92 Mbps achieved by BEContRA. Since RAGTS does not take into consideration the current channel condition, it does not look for a higher datarate after it stabilizes to a datarate, even when the channel condition improves. RASWTS performs better than the proposed ContRA algorithms at certain times. However, the proposed ContRA algorithms outperform it for most of the simulation duration. The overall throughput achieved by RASWTS in the case of UDP applications is 670.55 Mbps.

In the TCP application case, the overall throughput is limited to a maximum of $450 \mathrm{Mbps}$. Thus, for all the RA algorithms the maximum achievable throughput is $450 \mathrm{Mbps}$. The proposed GeContRA algorithm outperforms RAGTS as RAGTS is not able to quickly adapt to the changing channel conditions. However, RAGTS performs better than BEContRA algorithm for the initial 20 seconds. Since the BEContRA algorithm considers only the highest datarates for certain channel conditions, the datarate with highest expected throughput might have a probability of success less than $\delta$. In this case, the datarate with highest probability of success is chosen. However, this datarate might not give the maximum throughput for that channel condition as compared to the other datarates, which might have lower probability of success but higher expected throughput. As a result, the BEContRA algorithm initially does not perform well. The overall throughput achieved by RAGTS in the case of TCP applications is 301.83 Mbps as compared to $356.77 \mathrm{Mbps}$ achieved by GeContRA and 287.54 Mbps achieved by BEContRA. Similar to the UDP application case, the RASWTS algorithm at certain times performs better than the ContRA algorithms, but the ContRA algorithms perform better for most of the simulation duration. The overall throughput achieved by RASWTS in the case of TCP applications is $254.46 \mathrm{Mbps}$. The ContRA algorithms 


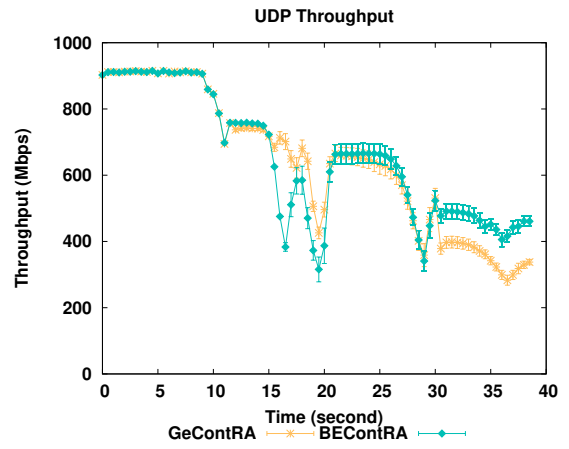

(a) Instantaneous UDP Throughput

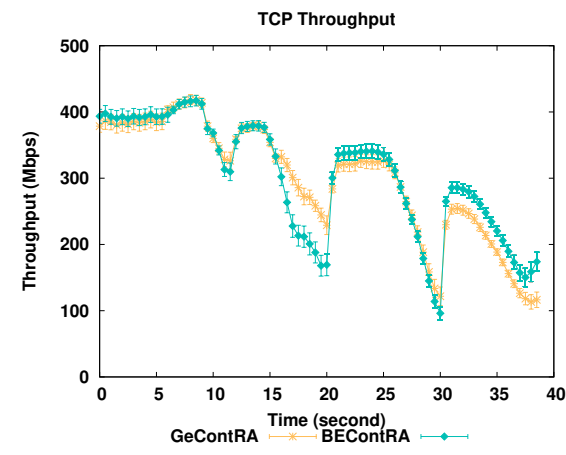

(b) Instantaneous TCP Throughput

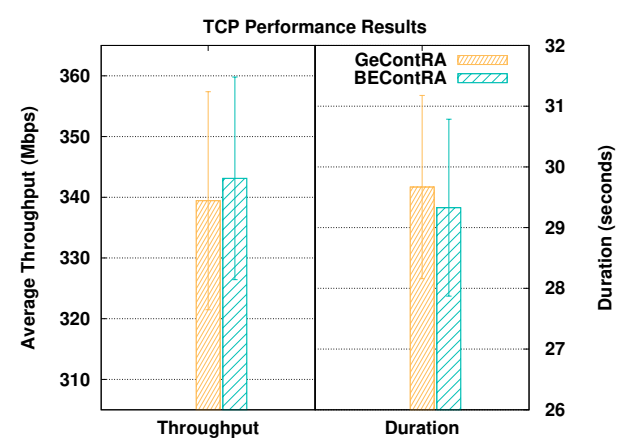

(c) TCP Performance Results

Fig. 5: Mobile device moving away from the AP with constant velocity in IEEE 802.11ax.

perform better than the other algorithms since it estimates the RSSI range the recipient is in and then chooses from the suitable datarate set. Thus, the overall performance of the ContRA algorithms is better than both the RAGTS and RASWTS algorithms.

Figure $4 \mathrm{c}$ presents the average throughput and average duration of a TCP application in IEEE 802.11ac when a mobile device is moving towards the AP. A total of $1.25 \times 10^{9}$ bytes is sent from the sender TCP application layer to the mobile device at one go. The confidence intervals are little bit high due to the same reasons as mentioned previously for the case of the mobile device moving away from the AP. In this case, all the RA algorithms take less time to send all the data as compared to the case where the mobile moves away from the AP. RAGTS takes the maximum time of 28.4 seconds, followed by RASWTS with 27.7 seconds. GeContRA and BEContRA takes a total 25.9 and 24.9 seconds respectively. Thus, GeContRA and BEContRA provide an improvement of $6.5 \%$ and $10.1 \%$ respectively over RASWTS and an improvement of $8.8 \%$ and $12.3 \%$ respectively over RAGTS in the overall duration. Similarly, GeContRA and BEContRA are able to achieve better throughput of 388.6 Mbps and 405.5 Mbps respectively as compared to 362.8 Mbps and 324.1 Mbps achieved by RAGTS and RASWTS respectively.

\section{B. Single mobile device in IEEE 802.11ax}

This section presents the performance evaluation results for rate adaptation algorithms in IEEE 802.11ax. The RAGTS and RASWTS algorithms are proposed only for IEEE 802.11ac standard. Thus, performance results of only the proposed ContRA algorithms are presented here. Similar type of configurations are used for the UDP and TCP applications as described in the previous section.

1) Mobile device moving away from the AP: Figures 5a and $5 \mathrm{~b}$ present the instantaneous UDP and TCP throughput in IEEE 802.11ax respectively when a mobile device moves away from the AP with constant velocity. The performances of both the versions of the ContRA algorithm are almost identical. For both UDP and TCP applications, the GeContRA algorithm performs better than the BEContRA algorithm between 15 seconds and 20 seconds and vice versa between 30 seconds and 40 seconds. As BEContRA chooses from a reduced rate set comprising only of the highest datarates for certain channel conditions, the datarate with highest expected throughput might have a probability of success less than $\delta$. In this case, the datarate with highest probability of success is chosen. However, this datarate might not give the maximum throughput for that channel condition as compared to the other datarates which are not included in the rate set. As a result, between 15 seconds and 20 seconds, the GeContRA algorithm is able to find a datarate which gives better throughput for that particular channel condition. For both the ContRA algorithms, the size of the datarate set increases as the channel condition deteriorates. However, the datarate set for the BEContRA algorithm is much smaller as compared to that of the GeContRA algorithm for the RSSI range below $-75 \mathrm{dBm}$. Thus, the BEContRA algorithm can easily and quickly find out the datarate which maximizes the throughput as compared to that of the GeContRA algorithm. That is why the BEContRA algorithm performs better than the GeContRA algorithm between 30 seconds and 40 seconds.

Figure $5 \mathrm{c}$ presents the average throughput and average duration of a TCP application in IEEE 802.11ax when a mobile device is moving away from the AP. The performances of both the GeContRA and BEContRA algorithms in IEEE 802.11ax are almost identical. The total duration for GeContRA and BEContRA is 29.7 and 29.3 seconds respectively and the throughput is $339.4 \mathrm{Mbps}$ and $343.1 \mathrm{Mbps}$ respectively.

2) Mobile device moving towards the AP: Figures 6a and $6 \mathrm{~b}$ present the instantaneous UDP and TCP throughput in IEEE 802.11ax respectively when a mobile device moves towards the AP with constant velocity. For both UDP and TCP, the proposed ContRA algorithms are able to adapt to improving channel condition. The performances of both the proposed algorithms are almost identical throughout, except for a few short periods. This happens due to the same reasons as mentioned above. Both the proposed algorithms are able to achieve high throughput by taking into consideration the RSSI range of the receiver and choosing from a rate set suitable for transmission in that RSSI range.

Figure $6 \mathrm{c}$ presents the average throughput and average 


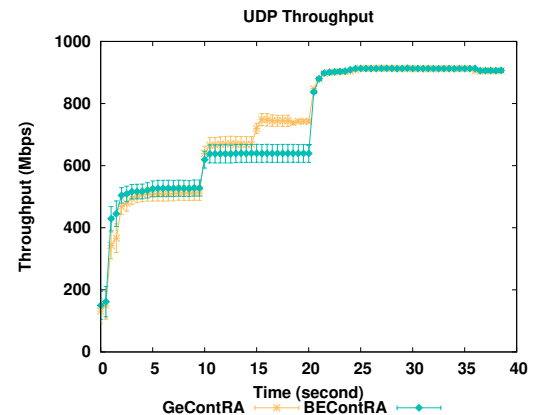

(a) Instantaneous UDP Throughput

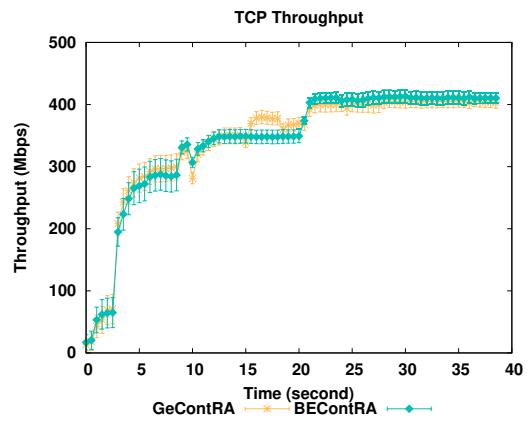

(b) Instantaneous TCP Throughput

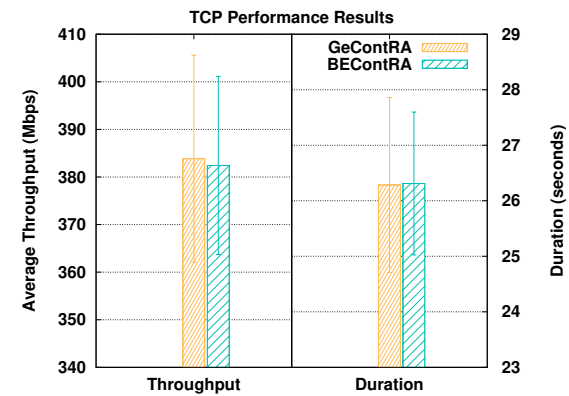

(c) TCP Performance Results

Fig. 6: Mobile device moving towards the AP with constant velocity in IEEE 802.11ax.

duration of a TCP application in IEEE 802.11ax when a mobile device is moving towards the AP. The performance of GeContRA and BEContRA in IEEE 802.11ax in the case of the mobile moving towards the AP is also better compared to the case of the mobile moving away from the AP. The total duration for GeContRA and BEContRA is 26.28 and 26.31 seconds respectively and the throughput is $383.9 \mathrm{Mbps}$ and 382.4 Mbps respectively.

\section{Performance Evaluation Results with multiple mobile users}

This section presents the performance evaluation results for the rate adaptation algorithms with multiple mobile users. The simulations are run with varying numbers of users $(5,10,15$, $20,25)$ connected to an AP. The overall traffic is fixed at $500 \mathrm{Mbps}$ and it is distributed equally among the users. For example, when there are 5 users, each user sends traffic at a constant rate of $100 \mathrm{Mbps}$ and when there are 25 users, each user sends traffic at a constant rate of $20 \mathrm{Mbps}$.

A mobile user uniformly chooses a direction and moves in that direction with a constant speed until it reaches the boundary. It again chooses another direction randomly and continues the same movement.

The simulations are run for both the proposed RA algorithms (GeContRA \& BEContRA) and RASWTS, as RASWTS is meant for mobility scenarios. Only UDP application is used for the performance evaluation, as the effect of the RA algorithms can be directly observed in the UDP throughput. This is not the case with TCP applications, as the TCP layer can also have a significant effect in the TCP throughput.

Figures $7 \mathrm{a}$ and $7 \mathrm{~b}$ present the average Normalized throughput and average Packet delivery percentage for varying number of users. The proposed RA algorithms are able to achieve high throughput (close to per user traffic) as well as high packet delivery percentage (above 90\%) for all the cases in both IEEE 802.11 ac and IEEE 802.11ax. On the other hand, for RASWTS both the throughput and packet delivery percentage reduces as the number of users increases. In fact, for the case of 25 users, RASWTS is able to achieve only 7.98 Mbps per user throughput as compared to $20 \mathrm{Mbps}$ per user traffic rate and $39.9 \%$ packet delivery percentage. This is because, for each receiver an independent instance of RASWTS is run, whereas both the proposed algorithms run a single instance for all the receivers and use the information learnt from other receivers for the choice of datarate. Thus, for the case of RASWTS, even if for a user a good datarate is determined for a certain channel condition, the same information has to be again determined for some other user. As a result, for each user every time there will be an initial period where the datarate is being determined for a certain channel condition. During this period, there will be packet loss if a sub-optimal datarate is chosen. In the case of the proposed algorithms, after a datarate is determined for a certain channel condition, the information can be used for all the other users later on. The algorithms do not determine the same datarate for a certain channel condition over and over again for different users. Thus, less packet loss and high throughput is achieved in the case of the proposed algorithms.

The GeContRA throughput for IEEE 802.11ax is lower than that of GeContRA for IEEE 802.11ac and BEContRA (for both IEEE 802.11ac and ax). The number of available datarates are higher in the case of 802.11ax. Thus, in general, the time taken to determine the best datarate will be higher as compared to that of 802.11ac RA algorithms, which may lead to higher packet loss in poor channel conditions. Thus, the GeContRA throughput for IEEE 802.11ax is lower as compared to that of GeContRA for IEEE 802.11ac.

The GeContRA algorithm uses all the datarates for an RSSI range, which will be able to successfully send packets in that range. In comparison, BEContRA algorithm uses only the highest datarates suitable for a particular RSSI range. Thus, the search space is reduced for the case of BEContRA and so determining the best datarate from the reduced set takes less time. Thus, throughput for GeContRA 802.11ac/ax is lower than that of BEContRA 802.11ac/ax respectively.

Figure $7 \mathrm{c}$ presents the average delay for varying number of users. The proposed RA algorithms experience much less delay as compared to the existing RASWTS algorithm. BEContRA experiences significantly less delay and the delay remains almost constant even with the increase in the number of users. Since less number of datarates for each RSSI range are considered, the convergence time for BEContRA is reduced, in turn reducing the overall packet delay. Though, the delay experienced by GeContRA fluctuates with the increase in the number of users it still remains below $100 \mathrm{~ms}$. Since 


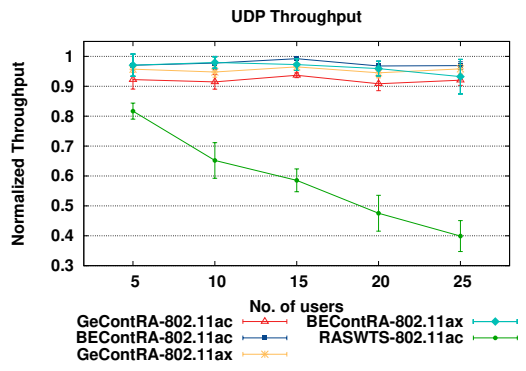

(a) Average Normalized Throughput

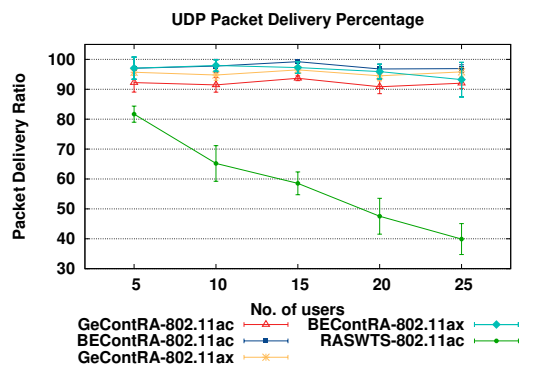

(b) Average Packet Delivery Ratio

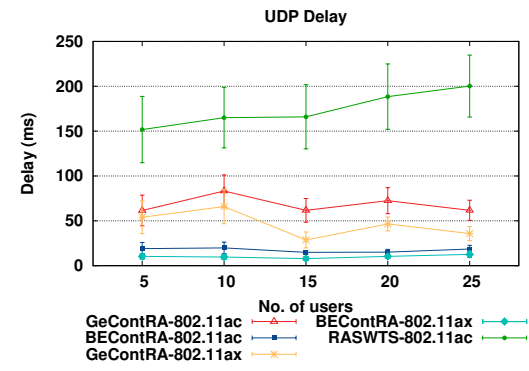

(c) Average Delay

Fig. 7: UDP Performance results for multiple mobile users.

GeContRA algorithm considers all the suitable datarates for a channel condition, the time taken to determine the optimal datarate for different channel conditions varies, thus resulting in fluctuating overall packet delay. Since BEContRA is able to achieve better overall throughput than that of GeContRA, the delay experienced is expectedly less for BEContRA. For RASWTS, the delay experienced is much higher as compared to that of BEContRA and GeContRA. RASWTS runs an instance of the RA algorithm per user whereas both GeContRA and BEContRA run a single instance of the RA algorithm for all the users. After an optimal rate is determined for a channel condition by both the GeContRA and BEContRA algorithms for a mobile user, the subsequent users will not face any additional delay for the same channel condition. For RASWTS algorithm, all the users will encounter the same initial delay of determining the optimal datarate, even though the same optimal datarate may have been already determined for some previous user. Thus, the delay experienced by RASWTS algorithm increases with the increase in the number of users. For 5 users, the delay experienced is $151.71 \mathrm{~ms}$ and for 25 users it increases to $200.25 \mathrm{~ms}$.

Both the proposed RA algorithms perform better than the RASWTS algorithm. There is hardly any performance deterioration of the proposed algorithms, whereas, the performance of the RASWTS algorithm deteriorates with the increasing number of users.

\section{CONCLUSiOnS}

In this paper, a Contextual Bandits based Rate Adaptation (ContRA) algorithm is proposed for mobile users in IEEE 802.11ac/ax. The RSSI range of the recipient is given as a context to the algorithm, and the algorithm tries to determine the optimal datarate from a rate set suitable for that RSSI range. Based on the type of datarate sets chosen for the RSSI ranges, two versions of the ContRA algorithm are proposed: GeContRA algorithm and BEContRA algorithm. Performance evaluation of the algorithms show that both of them are able to outperform existing RA algorithms and achieve high throughput for IEEE 802.11ac/ax. In the future, we plan to extend the ContRA algorithms for a Software Defined Networking (SDN) based WLAN comprising of multiple APs.

\section{REFERENCES}

[1] H. Gupta, A. Eryilmaz, and R. Srikant, "Low-Complexity, Low-Regret Link Rate Selection in Rapidly-Varying Wireless Channels," in Proc. of IEEE INFOCOM, 2018, pp. 540-548.

[2] R. Combes, J. Ok, A. Proutiere, D. Yun, and Y. Yi, "Optimal Rate Sampling in 802.11 Systems: Theory, Design, and Implementation," IEEE Transactions on Mobile Computing, vol. 18, no. 5, pp. 1145-1158, 2018.

[3] H. Gupta, A. Eryilmaz, and R. Srikant, "Link Rate Selection using Constrained Thompson Sampling," in Proc. of IEEE INFOCOM, 2019, pp. 739-747.

[4] H. Qi, Z. Hu, X. Wen, and Z. Lu, "Rate Adaptation with Thompson Sampling in 802.11ac WLAN," IEEE Communications Letters, 2019.

[5] R. S. Sutton and A. G. Barto, Reinforcement learning: An introduction. MIT Press, 2011.

[6] S. Jang, K. G. Shin, and S. Bahk, "Post-CCA and reinforcement learning based bandwidth adaptation in 802.11ac networks," IEEE Transactions on Mobile Computing, vol. 17, no. 2, pp. 419-432, 2017.

[7] L. Kriara and M. K. Marina, "SampleLite: A hybrid approach to $802.11 \mathrm{n}$ link adaptation," ACM SIGCOMM Computer Communication Review, vol. 45, no. 2, pp. 4-13, 2015.

[8] A. Sen and K. M. Sivalingam, "Rate Adaptation Techniques Using Contextual Bandit Approach for Mobile Wireless LAN Users," in Proc. of IEEE LCN, Nov. 2020, pp. 1-4.

[9] L. Zhou, "A survey on contextual multi-armed bandits," Feb. 2016. [Online]. Available: arxiv.org/abs/1508.03326

[10] D. Bouneffouf and I. Rish, "A survey on practical applications of multi-armed and contextual bandits," Apr. 2019. [Online]. Available: arxiv.org/abs/1904.10040

[11] IEEE, "Wireless LAN Medium Access Control (MAC) and Physical Layer (PHY) Specifications-Amendment 4: Enhancements for Very High Throughput for Operation in Bands below 6 GHz." IEEE Std 802.11ac-2013, pp. 1-425, Dec 2013.

[12] —, "Wireless LAN Medium Access Control (MAC) and Physical Layer (PHY) Specifications Amendment Enhancements for High Efficiency WLAN," IEEE P802.11ax/D3.0, June 2018, pp. 1-682, July 2018.

[13] "ns3: a discrete-event network simulator for Internet systems," Jun. 2020. [Online]. Available: www.nsnam.org

[14] "802.11ac MCS rates - Cisco Community," Jun. 2020. [Online]. Available: community.cisco.com/t5/wireless-mobility-documents/ 802-11ac-mcs-rates/ta-p/3155920

[15] M. Lacage, M. H. Manshaei, and T. Turletti, "IEEE 802.11 rate adaptation: a practical approach," in Proc. of ACM MSWIM, 2004, pp. $126-134$.

[16] S. H. Wong, H. Yang, S. Lu, and V. Bharghavan, "Robust rate adaptation for 802.11 wireless networks," in Proc. of ACM Mobicom, 2006, pp. $146-157$.

[17] S. Biaz and S. Wu, "Loss differentiated rate adaptation in wireless networks," in Proc. of IEEE Wireless Communications and Networking Conference (WCNC), 2008, pp. 1639-1644.

[18] Madwifi, "Minstrel rate adaptation algorithm," 2020. [Online]. Available: sourceforge.net/p/madwifi/svn/HEAD/tree/madwifi/trunk/ath_rate/ minstrel/minstrel.txt

[19] F. Fietkau, "Minstrel HT: New Rate Control Module for 802.11n," lwn. net/Articles/376765/, 2010. 
[20] S. Agrawal and N. Goyal, "Thompson sampling for contextual bandits with linear payoffs," in Proc. of International Conference on Machine Learning (ICML), 2013, pp. 127-135.

[21] O. Chapelle and L. Li, "An Empirical Evaluation of Thompson Sampling," in Proc. of International Conference on Neural Information Processing Systems. Curran Associates Inc., 2011, pp. 2249-2257.

[22] S. Agrawal and N. Goyal, "Analysis of Thompson Sampling for the Multi-armed Bandit Problem," in Proc. of the Annual Conference on Learning Theory. PMLR, 2012, pp. 39.1-39.26.

Arkadeep Sen received his Ph.D. degree in the Department of Computer Science and Engineering, Indian Institute of Technology, Madras. His research interests include Wireless Networking, Software Defined Networking and Network Function Virtualization.

Krishna M. Sivalingam is a Professor in the CSE Department, IIT Madras, Chennai, INDIA. Previously, he had held faculty positions at University of North Carolina, Greensboro; Washington State University, Pullman; and University of Maryland, Baltimore County. He is an IEEE Fellow, INAE Fellow and ACM Distinguished Scientist. 